\title{
Fullerenes with Heptagonal Rings An Overview
}

\author{
F. J. Sánchez-Bernabe \\ Departamento de Matemáticas \\ Universidad Autónoma Metropolitana Iztapalapa \\ 09340 México D F, Mexico \\ e-mail: fjsb@xanum.uam.mx
}

\begin{abstract}
Several works on nonclassical fullerenes with heptagons have mainly considered the case with just one heptagon. We present several nonclassical fullerenes with pentagons, hexagons and two, three, or more heptagons.
\end{abstract}

Keywords- Fullerene, heptagon, pentagon adjacency penalty rule, Euler's polyhedron formula.

\section{INTRODUCTION}

Classical fullerenes are those carbon cage molecules with exactly 12 pentagons and $n / 2-10$ hexagons, Fowler and Manolopoulos [1]. All classical fullerenes satisfy the so called isolated pentagon rule IPR, Kroto [2]. On the other hand, nonclassical fullerenes are those carbon cage molecules embedded with one or more squares or heptagons. In this last case, sometimes pentagon-pentagon adjacencies appear, and the most stable structure allows for the least pentagon-pentagon adjacencies, which is known as the pentagon adjacency penalty rule PAPR, Campbell et al [3].

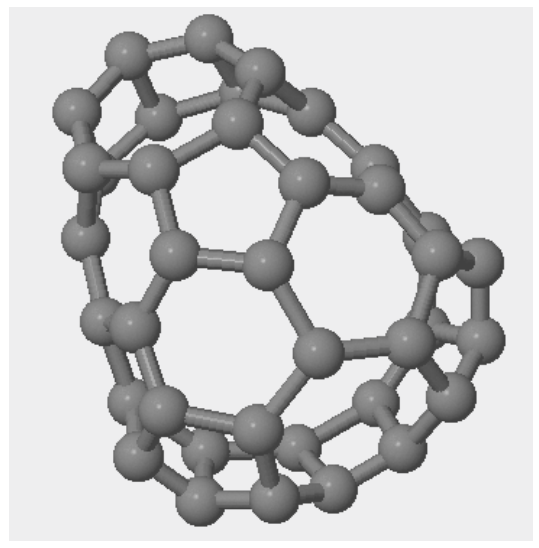

Figure 1. Molecule With 38 Carbons, 2 Heptagons, 4 HeXagons, AND 7 PENTAGONS, AND 7 SQUARES.

\section{BACKGROUND}

An amount of work has been done to study nonclassical fullerenes. For instance, Andrés Ayuela et al [4] show theoretical evidence for the existence of a nonclassical fulle-

\author{
Javier Salcedo \\ ESIME Culhuacán \\ Instituto Politécnico Nacional \\ 04430 México D F, Mexico \\ e-mail: jasr1240@gmail.com
}

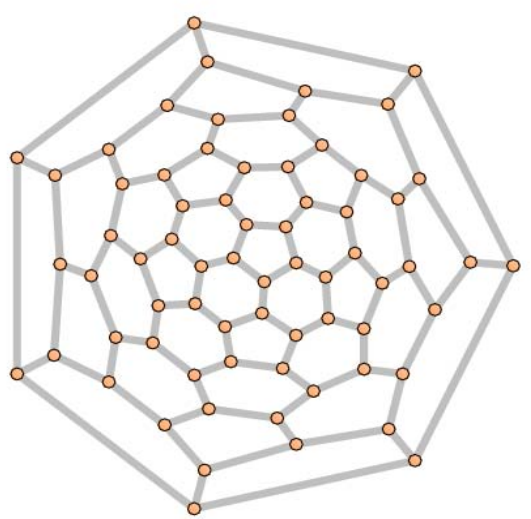

FIGURE 2. SCHLEGEL DiAGRAM FOR THE 68 CARBONS FULLERENE.

rene $\mathrm{C}_{62}$ with one heptagonal, 13 pentagonal and 19 hexagonal rings. Jie An et al [5] study the isomers of fullerene $\mathrm{C}_{26}$ composed of square, pentagonal, hexagonal, and heptagonal faces. Yuan-Zhi Tan et al [6] consider the fullerene $\mathrm{C}_{68}$ which contains one heptagonal ring. Furthermore, Li-Hua Gan et al [7] study fullerenes $\mathrm{C}_{46}$, $\mathrm{C}_{48}, \mathrm{C}_{50}$, and $\mathrm{C}_{52}$, some of them composed of one heptagonal ring.

\section{CALCULATIONS}

We have obtained the graphs of our results by running the V0.3 version of (Carbon Generator) CaGe software, Gunnar Brinkmann [8]. Schlegel diagrams are also provided for each considered fullerene.

Raghavachari et al [9] consider some corannulene-like form (bowl) of carbon molecules. Thus, inspired by that models, we present a preliminary example on Figure 1 with 38 carbons, 20 faces, and 56 edges.

We can observe in Figure 1 that, as it was mentioned previously besides heptagons, some nonclassical fullerenes may contain squares, and even octagons: Xian-Lei Sheng et al [10].

Our first real example of a nonclassical fullerene contains 68 carbons with two heptagonal rings, 14 pentagons, and 20 he- xagons. Therefore, this polyhedron 
has a total of 36 faces, 102 edges, and of course 68 vertexes.

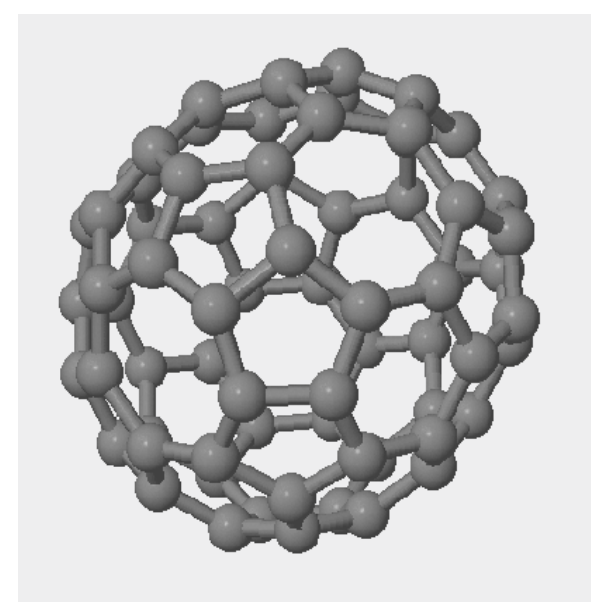

Figure 3. Fullerene With 68 Carbons, 2 Heptagons, 20 HeXagons, AND 14 PENTAGONS.

It is shown the 2-dimensional (Schlegel diagram) of this fullerene, and its 3-dimensional graph in Figure 2 and 3 , respectively.

The second example of nonclassical fullerene has 80 carbons, three heptagonal rings, 15 pentagons, and 24 hexagons. This polyhedron has a total number of 42 faces, 120 edges, and of course 80 vertexes. The Schlegel diagram of this fullerene is shown in Figure 4, and its 3dimensional graph in Figure 5.

Next, the third example of nonclassical fullerene contains 82 carbons with four heptagonal rings, 16 pentagons, and 23 hexagons. Thus, this polyhedron has a number of 43 faces, 123 edges, and of course 82 vertexes. It is shown the 2-dimensional representation of this fullerene in Figure 6, and its 3-dimensional graph in Figure 7.

The fourth example of nonclassical fullerene contains 76 carbons with six heptagonal rings, 18 pentagons, and 16 hexagons. Therefore, this polyhedron has a total number of 40 faces, 114 edges, and of course 76 vertexes. The 2dimensional representation of this fullerene is shown in Figure 8, and its 3-dimensional graph in Figure 9.

Finally, the fifth example of nonclassical fullerene contains 64 carbons with five heptagonal rings, 17 pentagons, and 12 hexagons. Therefore, this polyhedron has a total number of 34 faces, 96 edges, and of course 64 vertexes. It is shown the 2-dimensional representation of this fullerene in Figure 10, and its 3-dimensional graph in Figure 11.

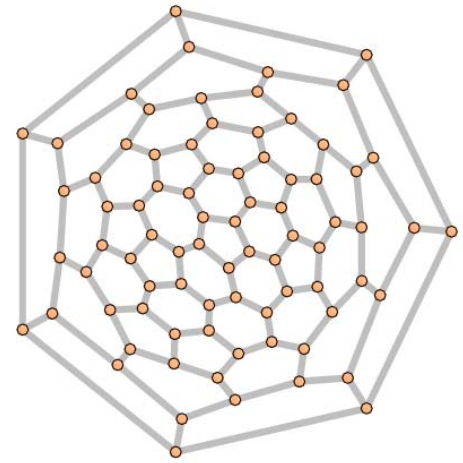

Figure 4. SCHLEgEl DiAgram For THE 80 CARBONS FULLERENE.

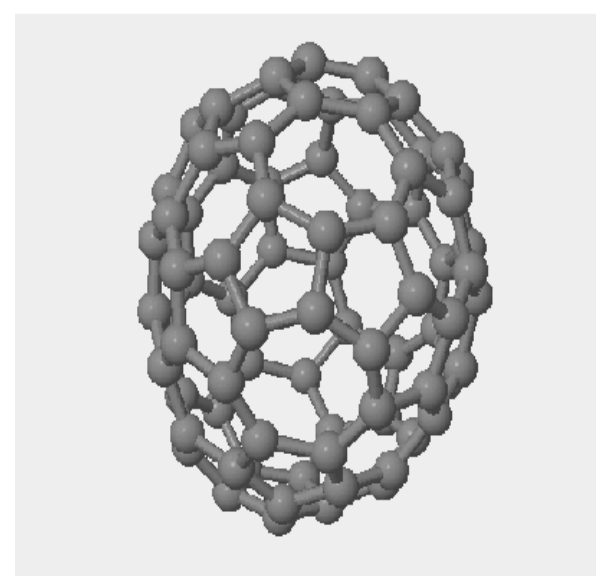

Figure 5. Fullerene With 80 CARBons, 3 Heptagons, 24HeXagons, AND 15 PENTAGONS.

\section{CONCLUSIONS}

We have considered five nonclassical fullerenes with two, three, four, five, and six heptagonal rings. In the case of the 68 carbons fullerene, it contains 2 heptagonal rings.

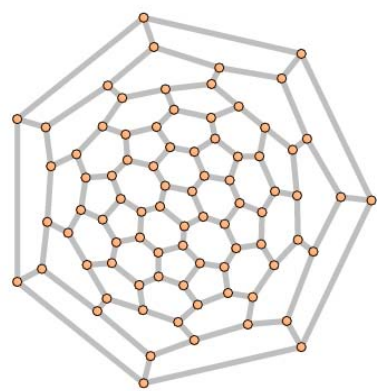

Figure 6. SCHLEgel Diagram FOR THE 82 CARBONS Fullerene.

One of them is surrounded by 4 pentagons next to each other, and one pentagon lies between 2 hexagons. 


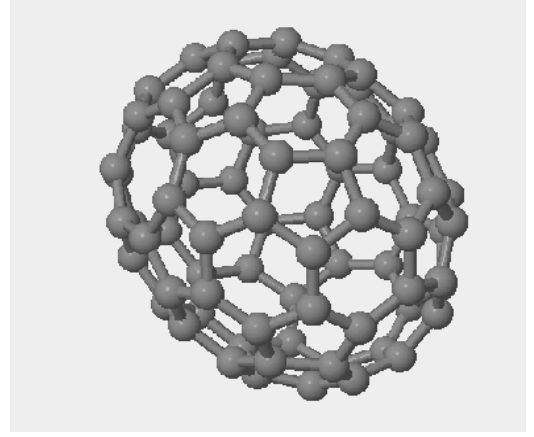

Figure 7. Fullerene With 82 CARbons, 4 Heptagons, 23HeXagons, AND 16 PENTAGONS.

The other heptagonal ring is surrounded by a couple of pentagons, which lies between two hexagons; the border is completed by one hexagon in the middle of 2 pentagons.

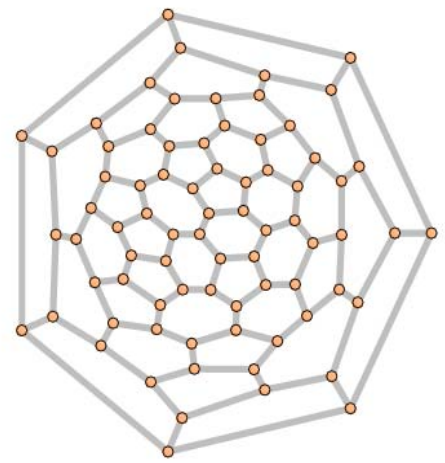

Figure 8. SCHLEgel DiAgRAm For the 76 CARBONS FULlERENE.

With respect to the 80 carbons case, the structure of the pentagons and hexagons surrounding each one of the 3 heptagonal rings follows one of the patrons of the 68 carbons fullerene.

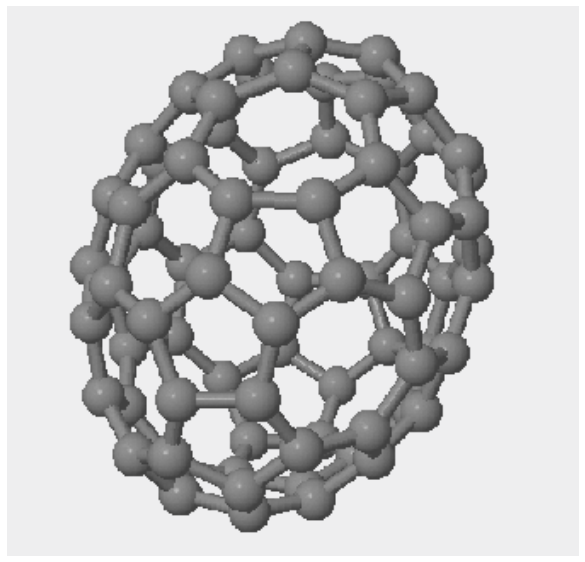

Figure 9. Fullerene With 76 Carbons, 6 Heptagons, 16 HeXagons, AND 18 PENTAGONS.

A similar situation occurs with the 82 carbons case. But, the 76 carbon fullerene, presents two new cases: a couple of pentagons, then a hexagon, followed by another couple of pentagons, and the boundary of this heptagonal ring is completed by 2 hexagons next to each other. The other type boundary surrounding a heptagonal ring is: a couple of hexagons, which lie between two pentagons; the border is completed by one pentagon in the middle of 2 hexagons.

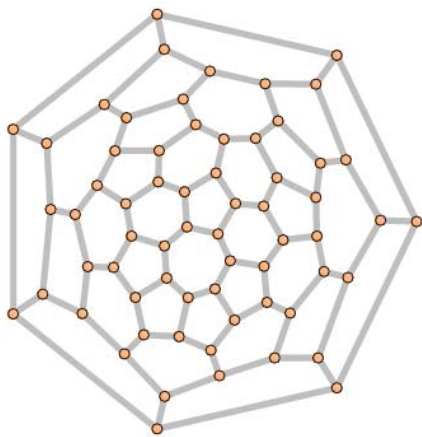

Figure 10. SCHLEgEl Diagram FOR THE 64 CARBONS FULLERENE.

Finally, another case appears on the 64 carbons fullerene: each one of two heptagonal rings are surrounded by 6 pentagons and only one hexagon.

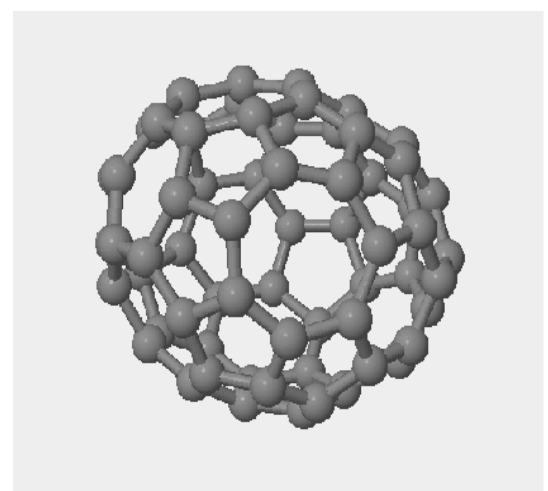

Figure 11. Fullerene With 64 CARbons, 5 Heptagons, 12 HEXAGONS, AND 17 PENTAGONS.

\section{REFERENCES}

[1] P. W. Fowler, and D. E. Manolopoulos D. E. An Atlas of Fullerenes. Oxford: Clarendon , 1995

[2] H. W. Kroto, "The stability of the fullerenes $C_{n}$ with $n=24,28,32$, 36, 50, 60 and 70" in Nature, vol. 329, pp. 529-531, 1986.

[3] E. E. B. Campbell, P. W. Fowler, D. Mitchell, and F. Zerbetto, "Increasing Cost of Pentagon Adjacency for Larger Fullerenes" in Chem. Phys. Lett.,vol. 250, pp 544-548, 1996

[4] A. Ayuela, P. W. Fowler, D. Mitchell, R. Schmidt, G. Seifert, and F. Zerbetto, " $\mathrm{C}_{62}$ : Theroretical Evidence for a Nonclassical Fullerene with a Heptagon Ring” in J. Phys. Chem., vol 100 pp 15634-36, 1996

[5] J. An, L.-H. Gan, J.-Q. Zhao, and R. Li, "A Global Search for the Lowest Energy Isomer of $C_{26}$ ” in J. Chem. Phys., vol 132, 154304, 2010.

[6] Y.-Z. Tan, Chen R.-T., Liao Z.-J., Li J., Zhu F., Lu X., Xie S.-Y., Li J., Huang R.-B., Zheng L.-S., "Carbon arc Production of 
Heptagon-Containing Fullerene[68]" in Nature Comm 2., vol 1431, 2011

[7] L.-H. Gan, Zhao J.-Q. and Hui Q, "Nonclassical Fullerenes with a Heptagon Violating the Pentagon Adjacency Penalty Rule", in J. Comp. Chem., vol. 31, 1715, 2010.

[8] G. Brinkmann and Dress A. W. M., "A constructive enumeration of fullerenes", in J. Algorithms, vol. 23, 345, 1997.
[9] K. Raghavachari, B. Zhang, J. A. Pope, B. G. Johnson, and P. M. W. Gill, "Isomers of $\mathrm{C}_{24}$. Density functional studies including gradient corrections", in Chem. Phys. Lett.,vol. 220, pp 385-390, 1994.

[10] X-L Sheng, H-J Cui, F. Ye, Q-B Yan, Q-R Shen, and G. Su, "Octagraphne as a versatile carbon atomic sheet for novel nanotubes, unconventional fullerenes, and hydrogen storage", in J. of Applied Phys., vol 112, 074515, 2012. 